\title{
Transseptal transcatheter mitral valve replacement in severe mitral annular calcification (transseptal valve-in-MAC)
}

\author{
Mayra Guerrero ${ }^{1}$, Mackram Eleid $^{1}$, Thomas Foley $^{2}$, Sameh Said $^{3}$, Charanjit Rihal ${ }^{1}$ \\ ${ }^{1}$ Department of Cardiovascular Medicine, ${ }^{2}$ Department of Radiology, ${ }^{3}$ Department of Cardiovascular Surgery, Mayo Clinic Hospital, Rochester, \\ MN, USA \\ Correspondence to: Mayra Guerrero, MD, FACC, FSCAI. Department of Cardiovascular Medicine, Mayo Clinic Hospital, 1216 2nd Street SW, \\ Rochester, MN, USA. Email: mayraguerrero@icloud.com; guerrero.mayra@mayo.edu.
}

Submitted Oct 09, 2018. Accepted for publication Oct 11, 2018.

doi: 10.21037/acs.2018.10.08

View this article at: http://dx.doi.org/10.21037/acs.2018.10.08

\section{Clinical vignette}

A 67-year-old female with Hodgkin's disease was treated with radiation therapy at age 17 , had chronic lymphocytic leukemia, breast cancer status post-bilateral mastectomies in 2000, partial nephrectomy, chronic kidney disease, deep venous thrombosis, pulmonary emboli on chronic anticoagulation, radiation-induced valvular heart disease, prior aortic valve replacement with a $21 \mathrm{~mm}$ Hancock II valve in 2009, pacemaker placement, chronic obstructive pulmonary disease on home oxygen therapy and moderate non-obstructive single vessel coronary artery disease with $60 \%$ stenosis of mid left anterior descending artery. She presented with symptomatic severe mitral stenosis and severe mitral annular calcification (MAC). She was New York Heart Association (NYHA) class III heart failure and transthoracic echocardiogram showed a mitral valve area of $1.2 \mathrm{~cm}^{2}$ with a mean mitral gradient of $11 \mathrm{mmHg}$. Although her Society of Thoracic Surgery predicted risk of mortality score for reoperation for mitral valve replacement alone was $6.49 \%$, she was considered to have extreme surgical risk due to her multiple medical comorbidities, porcelain aorta, hostile chest and the presence of severe MAC. She was referred for possible transcatheter mitral valve replacement (TMVR).

\section{Pre-procedural planning}

A cardiac computed tomography (CT) TMVR protocol was obtained as per routine in the evaluation of these patients. She was found to have high risk of TMVRinduced left ventricular outflow tract (LVOT) obstruction. Her mitral annular area was $680 \mathrm{~mm}^{2}$ and using a $29 \mathrm{~mm}$ virtual SAPIEN 3 valve, the predicted Neo-LVOT area was $138 \mathrm{~mm}^{2}$, which is considered high risk for LVOT obstruction. For that reason, and considering she had favorable anatomy for alcohol septal ablation, the structural heart disease team decided to recommend alcohol septal ablation 3 to 4 weeks prior to TMVR to decrease the risk of TMVR-induced LVOT obstruction. She underwent uneventful alcohol septal ablation with $1 \mathrm{~mL}$ of dehydrated alcohol injected in the first septal perforator branch over 5 min. Repeat cardiac CT 3 weeks after alcohol septal ablation showed favorable remodeling of the basal septum with a new predicted Neo-LVOT area of $217 \mathrm{~mm}^{2}$. The decision was to proceed with transeptal TMVR in MAC.

\section{Procedural technique}

The TMVR procedure was performed in the cardiac catheterization laboratory under general anesthesia and transesophageal echocardiogram (TEE). A cardiac CT scan was used to determine the transeptal location and fluoroscopic deployment angle. Left femoral arterial access was obtained with a $45 \mathrm{~cm} 6$ French (Fr) sheath. A long sheath is used to allow simultaneous left ventricular and aortic pressure monitoring during the procedure through a $5 \mathrm{Fr}$ pigtail catheter and the side arm of the $6 \mathrm{Fr}$ long sheath in descending aorta. A 7 Fr sheath was placed in the left femoral vein. Right femoral venous access was obtained with 7 Fr sheath and the site was pre-closed with one Proglide closure device (Abbott Vascular, Abbott Park, IL). A 5 Fr temporary transvenous pacemaker was placed in the right ventricle via the left femoral vein. A 5 Fr pigtail catheter was placed in the left ventricle through the sheath 
in left femoral artery for simultaneous left ventricular and aortic pressure monitoring. The sheath in the right femoral vein was upsized to a 16 Fr Edwards e-Sheath. Transeptal puncture was performed with a 7 Fr Mullins sheath and a BRK needle (St. Jude Medical, St. Paul, MN) through the e-Sheath under fluoroscopic and TEE guidance. A $230 \mathrm{~cm}$ 0.025 inch Toray wire was introduced in the left atrium, the Mullens sheath was removed and a 14 Fr Toray dilator was used to dilate the interatrial septum. The dilator was removed and a 9.5 Fr Dexterity deflectable sheath (Spirus Medical, LLC, Bridgewater, MA) was placed in the left atrium. Another deflectable sheath that can be used for this purpose is the $8.5 \mathrm{Fr}$ Agilis steerable introducer (St. Jude Medical, St. Paul, MN). The Dexterity sheath was flexed to aim towards the mitral valve. The mitral valve was crossed with a $6 \mathrm{Fr}$ pigtail catheter and a J wire through the Dexterity sheath. The J wire is left inside the pigtail to provide support but it is not used to cross the valve with wire alone. The intention is to avoid wire entanglement through chordae, such risk is lower when crossing the valve with the pigtail catheter itself. Once the pigtail is in the left ventricle, a 0.035 extra small Safari wire (Boston Scientific, Marlborough, MA, USA) was introduced in the left ventricle and positioned at the apex. The pigtail catheter was removed and a $14 \mathrm{~mm}$ Tyshak balloon was introduced to perform septostomy. After septostomy is completed, the partially inflated Tyshak balloon was advanced across the mitral valve to "floss" to test the trajectory and freedom from chordae in the subvalvular apparatus. The balloon was removed and a $29 \mathrm{~mm}$ SAPIEN 3 valve (Edwards Lifesciences, Irvine, CA, USA) prepared with $5 \mathrm{~mL}$ of additional contrast was introduced in the IVC. The SAPIEN 3 valve must be mounted with the sealing skirt towards the left atrium and the open cells towards the left ventricle. Valve alignment over the balloon delivery system is performed as is done for TAVR in the $\mathrm{AP}$ projection with cranial angulation if needed to remove parallax to visualize stent frame in long axis. Then, the delivery system may be rotated counterclockwise 90 to 180 degrees over its own axis to have the Edwards logo facing the operator or facing down. This facilitates flexing the Edwards system towards the mitral valve in the opposite direction as is done for TAVR. The system is then advanced slowly across the interatrial septum and the mitral valve. If the system cannot be advanced across the mitral valve because the pusher cannot cross the interatrial septum, the pusher can be unlocked and the balloon delivery system advanced across the mitral valve independent of the pusher (i.e., telescoping). Once the system is across the mitral valve, the flex tube/pusher is unlocked and pulled back until it meets the most distal of the 3 radio-opaque markers in the delivery system. Pulling more than that may result in the flex tube pulled back to the right atrium which would not be helpful and may decrease the operator's ability to push and advance the system during deployment. The fluoroscopy $\mathrm{C}$-arm is then positioned in the pre-determined deployment angle. If the landing zone of the ventricular edge of the stent has been determined by CT analysis, i.e., by finding a piece of calcium or other radio-opaque marker, the ventricular edge of the stent is placed at such landing zone. The valve is then deployed slowly under rapid pacing (140 to $180 \mathrm{bpm}$ ). A slow deployment facilitates adjustment of position if needed. Once deployed, the balloon is deflated and pulled back into the left atrium and the performance of the mitral prosthesis is evaluated by TEE.

There was no LVOT obstruction detected by TEE or hemodynamic catheterization measurements. Moderate to severe paravalvular leak (PVL) was noted on TEE at the commissures, which was anticipated based on large intercommissural diameter by cardiac CT of $34.7 \mathrm{~mm}$. The decision was to proceed with PVL closure. The PVL defect was crossed with a wire but we were unable to cross with catheter to deliver a closure device. Therefore, the decision was to perform balloon post-dilatation with the same Edwards balloon adding additional contrast volume. Post-dilatation with 3 additional $\mathrm{mL}$ of contrast (total $8 \mathrm{~mL}$ additional) resulted in partial improvement of the leak. Additional post-dilatation with 2 more $\mathrm{mL}$ of contrast (total 10 additional $\mathrm{mL}$ ) reduced the PVL to moderate. The balloon catheter and sheaths were removed. Hemostasis was achieved in the right femoral vein with the previously placed Perclose suture, in the left femoral artery with Angioseal closure device and the left femoral vein with manual pressure. Intra-procedural TEE at end of the procedure showed moderate PVL, a mean mitral gradient of $1 \mathrm{mmHg}$ and no LVOT obstruction. Transthoracic echocardiogram the following day showed mean mitral gradient of $2 \mathrm{mmHg}$, mean LVOT gradient of $3 \mathrm{mmHg}$, trace central mitral regurgitation and trace PVL. At 30-day follow-up, the patient reported significant improvement of symptoms, tolerating cardiac rehabilitation therapy without dyspnea on exertion (NYHA I). Transthoracic echocardiogram at 30 days showed preserved left ventricular ejection fraction, trace to mild PVL, mean mitral gradient of $5 \mathrm{mmHg}$ and mean LVOT gradient of $2 \mathrm{mmHg}$. 


\section{Comments}

Transcatheter mitral valve replacement with balloonexpandable valves in patients with severe MAC is associated with high risk of LVOT obstruction (1). Percutaneous alcohol septal ablation has been used as a bail-out strategy to reduce LVOT gradient when severe LVOT obstruction with hemodynamic compromise occurs after transseptal TMVR $(2,3)$. However, prevention of LVOT obstruction is preferred and highly recommended to improve outcomes. Operators can identify patients at high risk of LVOT obstruction by evaluating the neo-LVOT area on cardiac CT analysis. The threshold neo-LVOT area at which obstruction occurs is not well understood at this time. One study found that a neo-LVOT area of $189 \mathrm{~mm}^{2}$ on cardiac CT or smaller was associated with LVOT gradients by cardiac catheterization (4). Patients with neo-LVOT areas of $189 \mathrm{~mm}^{2}$, or smaller, may benefit from LVOT obstruction risk reduction strategy.

One option to reduce LVOT obstruction risk is to surgically remove the anterior leaflet during open transatrial TMVR (5). However, not all patients are good candidates for such an invasive option. Percutaneous laceration of the anterior leaflet is another alternative (6), but the procedure is challenging and may not always be effective. Preemptive alcohol septal ablation performed 3 to 4 weeks prior to TMVR has been successfully used to reduce the risk of LVOT obstruction in patients with adequate anatomy (7). Although this option is not free of risk, we have found reproducible results that facilitate safe transeptal TMVR as demonstrated in this case.

\section{Advantages}

The main advantage is that alcohol ablation is a procedure many interventional cardiologists are familiar with it and it can be performed without additional extensive training or special equipment.

\section{Caveats}

The most important caveat is that patients require two separate procedures, one for alcohol ablation and the second one for TMVR weeks later. Not all patients are stable enough to wait this period of time. In addition, not all patients have favorable anatomy. Furthermore, alcohol septal ablation carries its own risk and may not always be effective at increasing neo-LVOT area enough to allow safe TMVR.

\section{Conclusions}

Percutaneous alcohol septal ablation allows safe transeptal TMVR with balloon-expandable valves in selected patients with severe MAC who have high risk of TMVR-induced LVOT obstruction.

\section{Acknowledgements}

None.

\section{Footnote}

Conflicts of Interest: Dr. Guerrero has served as a proctor and consultant and has received research grant support from Edwards Lifesciences. Other authors have no conflicts of interest to declare.

\section{References}

1. Guerrero M, Urena M, Himbert D, et al. 1-Year Outcomes of Transcatheter Mitral Valve Replacement in Patients With Severe Mitral Annular Calcification. J Am Coll Cardiol 2018;71:1841-53.

2. Guerrero M, Wang DD, O'Neill W. Percutaneous alcohol septal ablation to acutely reduce left ventricular outflow tract obstruction induced by transcatheter mitral valve replacement. Catheter Cardiovasc Interv 2016;88:E191-7.

3. Guerrero M, Wang DD, Himbert D, et al. Short-term results of alcohol septal ablation as a bail-out strategy to treat severe left ventricular outflow tract obstruction after transcatheter mitral valve replacement in patients with severe mitral annular calcification. Catheter Cardiovasc Interv 2017;90:1220-6.

4. Wang DD, Eng MH, Greenbaum AB, et al. Validating a prediction modeling tool for left ventricular outflow tract (LVOT) obstruction after transcatheter mitral valve replacement (TMVR). Catheter Cardiovasc Interv 2018;92:379-87.

5. Russell HM, Guerrero ME, Salinger MH, et al. Open Atrial Transcatheter Mitral Valve Replacement in Patients With Mitral Annular Calcification. J Am Coll Cardiol 2018;72:1437-48.

6. Babaliaros VC, Greenbaum AB, Khan JM, et al. Intentional Percutaneous Laceration of the Anterior Mitral Leaflet to Prevent Outflow Obstruction During Transcatheter Mitral Valve Replacement: First-in-Human Experience. JACC Cardiovasc Interv 2017;10:798-809. 
7. Guerrero M. 30-Day Outcomes of Transcatheter Mitral Valve Replacement in Patient With Severe Mitral Valve Disease Secondary to Mitral Annular Calcification of Failed Annuloplasty Rings. Data presented as Late Breaking Clinical Trial at Transcatheter Cardiovascular

Cite this article as: Guerrero M, Eleid M, Foley T, Said S, Rihal C. Transseptal transcatheter mitral valve replacement in severe mitral annular calcification (transseptal valve-in-MAC). Ann Cardiothorac Surg 2018;7(6):830-833. doi: 10.21037/ acs.2018.10.08
Therapeutics Scientific Symposium 2017. Available online: https://www.tctmd.com/slide/30-day-outcomestranscatheter-mv-replacement-patients-severe-mitralvalve-disease-secondary 